Jaromír KADLEC

Université Palacký d'Olomouc

\title{
PARTICULARITÉS LEXICALES DU FRANÇAIS AU BURUNDI
}

\section{Situation géographique}

Le Burundi est un petit pays situé en Afrique centrale dans la région des Grands Lacs. La superficie du pays est de $27834 \mathrm{~km}^{2}$. Le Burundi est limité à l'ouest par la République démocratique du Congo, au nord par le Rwanda, donc par des États qui ont subi, avec le Burundi, la domination coloniale belge. À l'est et au sud on trouve la Tanzanie, ancienne colonie britannique ayant pour langues officielles l'anglais et le swahili. ${ }^{1}$

\section{Histoire}

À partir de la fin du XIX ${ }^{\mathrm{e}}$ siècle, le Burundi et le Rwanda ont fait partie de l'Afrique-Orientale allemande. De 1916 à 1962, les deux pays ont été sous mandat, puis sous tutelle belge. Le Burundi est devenu indépendant en 1962. La vie politique du Burundi et du Rwanda est dominée par des rivalités qui opposent les Hutu, majoritaires dans les deux pays, et les Tutsi qui représentent environ $14 \%$ de la population du Burundi. On peut mentionner les massacres de 1972 et de 1988 au Burundi, ou le génocide de 1994 au Rwanda, déclenché par la mort des présidents burundais et rwandais dans un avion militaire offert par la France et piloté par un équipage français, qui a été abattu près de Kigali.

\section{Population et situation linguistique}

La population du Burundi est estimée à 6,6 millions d'habitants. La plupart des Burundais vivent en zone rurale. Le taux d'urbanisation dans le pays est l'un des plus faibles au monde $(10 \%)$. Tous les Burundais d'origine parlent la même langue, le kirundi, une langue bantoue très proche du kinyarwanda, langue parlée par tous les Rwandais. Les Hutu et les Tutsi parlent donc la même langue et la distinction est plutôt sociale qu'ethnique. ${ }^{2}$

Avant 1992, le français et le kirundi étaient considérés comme les langues officielles du Burundi. Selon l'article 10 de la Constitution de 1992, les langues officielles sont le kirundi et les autres langues déterminées par la loi. L'article 5 de la Constitution de 2004 reprend les dispositions de la Constitution de 1992, et ajoute que tous les textes législatifs doivent avoir leur version originale en kirundi. Si l'on s'en tient strictement aux dispositions constitutionnelles, l'officialité n'est reconnue qu'au kirundi. Cependant, le français demeure de facto l'une des deux langues officielles.

\footnotetext{
${ }^{1}$ Le swahili a le statut de langue officielle aussi au Kenya.

${ }^{2}$ L'administration coloniale belge a exigé que tout propriétaire de dix vaches et plus soit considéré comme un Tutsi et les autres sont devenus automatiquement des Hutu.
} 


\section{Particularismes lexicaux du français au Burundi}

Plusieurs dénominations spécifiques désignent les espèces végétales qui croissent dans la région des Grands Lacs, la faune et les produits gastronomiques locaux. Parmi les termes désignant les plantes, on peut citer éleusine « céréale estafricaine Eleusine coracana, adaptée aux altitudes élevées» ou ensete (faux bananier) «variété de bananier qui pousse à l'état sauvage au Burundi ». ${ }^{3}$

Assez nombreux sont les particularismes qui désignent les poissons. On peut mentionner par exemple koué ou kouhé (origine inconnue) "poisson Bulengerochromis microlepi de la famille des cichlidés, que l'on trouve fréquemment dans le lac Tanganyika », mukeke (origine inconnue) «poisson prédateur Luciolates stappersii du Lac Tanganyika », sangala (probablement un emprunt aux langues congolaises) « gros poissons prédateurs Lates angustifrons et Lates mariae du Tanganyika ». Kimputu (emprunt au swahili kimputu « arachnidée Ornithodoros moubata de la famille des argasides ») est une tique qui transmet une fièvre récurrente, le substantif mamba (emprunt au kirundi imamba «vipère ») désigne un serpent venimeux mesurant de 2,5 à 4 mètres dont la morsure est mortelle.

Le français employé au Burundi est riche en dénominations désignant les produits gastronomiques locaux. Parmi les mots attestés et fréquemment employés, on peut citer bugali (emprunt au kirundi ubugali ou ubugari «pâte ») «pâte préparée avec de la farine de manioc et présentée sous forme de boule », imbabura ou imbabula (emprunt au kirundi imbabura < swahili «brasero ») «brasero de fabrication artisanale », insongo (emprunt au kirundi insongo «bière alcoolisée ») «boisson alcoolisée obtenue par la fermentation de jus de banane très concentré, additionné d'une très petite quantité d'eau », michopo (emprunt au swahili chopo «nourriture ») «plat d'origine ouest-africaine, à base de viande fumée et assaisonnée », mwambe ou moambe (probablement un emprunt aux langues congolaises) «plat à base de poulet, de sombe, de bananes et de riz », rutuku (emprunt au kirundi urutuku «couleur rouge ») «boisson fortement alcoolisée obtenue par la distillation de pâtes de manioc et de maïs mélangées avec de l'eau », urwarwa (emprunt au kirundi urwarwa ou inzarwa «bière de bananes ») «bière de bananes obtenue par fermentation du jus de bananes et additionnée de $50 \%$ d'eau ».

Certaines particularités décrivent les instruments de musique : inanga (emprunt au kirundi inanga « cithare heptacorde en auge ») « sorte de cithare, dont la corde unique est tendue de 6 à 9 fois de haut en bas sur un plateau de bois creusé et servant de bac de résonance », indonongo (emprunt au kirundi idonongo) «grand violon à une corde, dont les caisses de résonance sont constituées de calebasses » et les vêtements : imvutano (emprunt au kirundi imvutano) « vêtement traditionnel composé de deux pièces, fait de tissu léger, dont le haut se noue sur une épaule».

Très nombreuses sont les particularités lexicales désignant les faits qui sont propres à la vie sociale et culturelle des Burundais. On peut mentionner par exemple les mots agasimbo (emprunt au kirundi agasimbo ou udusimbo "danse folklorique du Burugane ») «danse acrobatique traditionnelle », igisoro (emprunt au kirundi igisoro ou ibisoro «emplacement destiné à jouer au trictrac ») « jeu à

\footnotetext{
${ }^{3}$ Les graines de l'ensete sont utilisées comme billes pour jouer à l'ikibuguzo.
} 
deux joueurs qui se joue sur une tablette de bois creusée de 32 cupules, dans lesquelles on déplace des billes » kibuguzo ou ikibuguzo (emprunt au kirundi ikibuguzo «genre de trictrac») « jeu à deux joueurs qui se joue sur une tablette de bois creusée de 32 cupules, dans lesquelles on déplace des billes constituées généralement de graines de faux-bananier », kubandwa (emprunt au kirundi $k u b a n d w a)$ «culte rituel ou initiatique pratiqué à des occasions importantes pour éloigner la maladie ou le malheur », kwasakwasa ou kwasa kwasa (emprunt aux langues congolaises) « danse d'origine congolaise ».

Le français au Burundi emprunte naturellement beaucoup de mots au kirundi. On peut citer par exemple les expressions ganwa (emprunt au kirundi umuganwa ou abaganwa «prince de sang ») « lignée princière de la population burundaise », hima (emprunt au kirundi abahima « groupe de lignages comprenant une trentaine de familles ») «clan tutsi au Burundi », hutukazi, umuhutukazi ou muhutukazi (emprunt au kirundi umuhutukazi ou abahutukazi «femme hutu ») «femme d'origine hutu », intore (emprunt au kirundi intore « cadet incorporé dans l'armée royale », «danseur») «danseur traditionnel », «danse exécutée par ces danseurs », itongo (emprunt au kirundi intongo ou amatongo «terrain», «propriété ») «parcelle de terrain dévolue à chaque paysan », kokayi (emprunt au kirundi umukokayi) «personne qui racole les voyageurs pour une destination donnée », makala (emprunt au kirundi ikara ou amakara «charbon », «braise ») «charbon de bois », тиfити ou тирfuтu (emprunt au kirundi итирfuти ou abapfumu «guérisseur ») « sorcier », muganuro (emprunt au kirundi umuganuro «fête des prémices », «fête des semailles du sorgho ») 《fête traditionnelle qui marquait le début des semailles du sorgho», ${ }^{4}$ murundi (emprunt au kirundi umurundi ou aburundi «habitant du pays Rundi ») «personne de nationalité burundaise », mushingantahe ou umushingantahe (emprunt au kirundi umushingantahe « magistrat», «notable», « conseiller», « arbitre ») «personne de sexe masculin ayant des responsabilités sociales, judiciaires ou administrastives », «personne investie de respect en raison de son âge, de sa moralité, de son savoir » (même si elle n'a aucune responsabilité administrative), muzungu ou umuzungu (emprunt au kirundi umuzungu «homme de race blanche ») «Européen », muzungukazi (emprunt au kirundi umuzungu « de race blanche » et kazi «femme») «Européenne», mwami (emprunt au kirundi umwami ou abami « roi ») «monarque de droit divin du Burundi traditionnel», rugo (emprunt au kirundi urugo ou ingo «enclos ») «ensemble entouré d'une clôture circulaire, composé de cases et cours intérieures, dans lequel vivent les personnes et les animaux domestiques », rusengo (emprunt au kirundi rusengo) « fontaine en maçonnerie », zаmu (emprunt au kirundi umunyezamu «gardien qui dort normalement couché près de l'habitation ou des marchandises qu'on lui confie ») «gardien qui surveille une maison, une voiture ou toute autre marchandise $»$.

Le français au Burundi est relativement riche en calques du kirundi : aller à Ijenda (calque du kirundi kuja mw'i Jenda) «se mettre à l'écart pour avoir une

\footnotetext{
${ }^{4}$ Le dernier muganuro, cérémonie importante du Burundi traditionnel, qui avait lieu au mois de décembre, et au cours de laquelle le mwami donnait l'autorisation de commencer les semailles du sorgho, fut célébré en 1929.
} 
conversation discrète », ${ }^{5}$ etre avec un ventre «être enceinte », prendre un ventre « tomber enceinte », se connaître avec qqn « connaître qqn ».

Certains mots ont été empruntés au kinyarwanda : inkotanyi (emprunt au kinyarwanda inkotanyi) « ceux qui se battent sans laisser de répit à l'adversaire », inyenzi (emprunt au kinyarwanda inyenzi « cancrelat») «membre de la branche armée des réfugiés rwandais de la première génération ». ${ }^{6}$

Outre le kirundi et le français, c'est également le swahili, langue véhiculaire importante de la région des Grands Lacs comptant le plus grand nombre de locuteurs de l'Afrique noire, ${ }^{7}$ qui est employé au Burundi et au Rwanda. Au Burundi, il est parlé surtout par les étrangers dans les villes et appris essentiellement en dehors du système formel, car l'Église catholique freine l'expansion du swahili, trop lié à l'islam.

Parmi les emprunts au swahili, langue africaine la plus enseignée dans le monde, on peut mentionner américani ou amérikani (emprunt au swahili amerikani «cotonnade blanche ») «cotonnade blanche », ${ }^{8}$ bilulu (emprunt au swahili kilulu, vilulu ou bilulu « insecte ») « insecte nuisible », bwana (emprunt au swahili bwana «personnage important») «patron, monsieur», kabisa (emprunt au swahili kabisa «complètement ») «beaucoup», kambambili (emprunt au swahili kamba «ficelle » et mbili «deux») «sandale fabriquée artisanalement, avec des matériaux de récupération», matata (emprunt au swahili matata «difficultés ») «problèmes », nyumbakumi (emprunt au swahili nyumba «maison» et kumi «dix») « chef de quartier», pili-pili (emprunt au swahili pilipili «poivre, piment») «piment rouge», « assaisonnement fabriqué avec le piment, sous forme de poudre ou de sauce piquante », sombe (emprunt au swahili sombe «feuilles de manioc, qui servent de légumes ») «plat constitué de feuilles de manioc pilées et cuites ».

Le français utilisé au Burundi a emprunté au kirundi aussi des mots d'origine swahili : barza (emprunt au kirundi ibaraza ou amabaraza < swahili ibaraza « véranda ») «terrasse », mbabula ou mbabura (emprunt au kirundi imbabura < swahili babula «petit brasero à charbon de bois servant à la cuisine ») «brasero de fabrication locale , rupango (emprunt au kirundi urupango ou impango < swahili lupango « enclos ») « maison qui donne sur la rue et dont l'arrière-cour est entourée de chambres à louer ».

Le mot soko «marché », qui est d'origine arabe, est passé dans le français au Burundi par l'intermédiaire du swahili et du kirundi (emprunt au kirundi isoko ou au swahili soko < arabe « souk »).

Les emprunts indirects d'origine lingala, langue très répandue dans la République démocratique du Congo, sont également attestés. On peut citer, par exemple, le substantif ikembe (emprunt au kirundi ikembe < lingala «sanza, boîte musicale à lamelles ») «instrument de musique en forme de petite caisse, sur laquelle sont fixées une douzaine de lamelles métalliques que le joueur fait vibrer avec le pouce et l'index ». Le mot mwambe «préparation culinaire à base de

\footnotetext{
${ }^{5}$ Les habitants de la région d'Ijenda ont la réputation d'une discrétion exagérée.

${ }^{6}$ Les réfugiés tutsi du Rwanda ont quitté leur pays suite aux troubles de 1959.

${ }^{7}$ On estime que 40 à 50 millions de locuteurs pratiquent le swahili comme langue maternelle ou (surtout) comme langue seconde.

${ }^{8}$ Le mot vient de l'importance prise au milieu du $\mathrm{XX}^{\mathrm{e}}$ siècle sur la côte orientale de l'Afrique par les importations des cotonnades de la Nouvelle-Angleterre.
} 
poulet, de sombe, de bananes et de riz » est également emprunté aux langues parlées dans la République démocratique du Congo.

La région des Grands Lacs subit les offensives politiques et linguistiques des pays anglophones ayant pour objectif, entre autres, d'y imposer la langue anglaise. La position et l'avenir du français au Burundi, au Rwanda et au Congo, pays traditionnellement francophones, sont menacés. Aujourd'hui, la Francophonie est assez mal perçue au Burundi et au Rwanda à cause du rôle de la France dans le génocide de 1994 au Rwanda et le français est considéré comme un «mal nécessaire », hérité du colonialisme, pour la communication avec l'extérieur. ${ }^{9}$ La percée actuelle de l'anglais dans les trois pays est également consécutive à l'image positive que la population a de cette langue. L'anglais a dans les anciennes colonies belges en Afrique l'image de langue d'une civilisation moderne, sans passé colonial. Les trois pays développent des relations politiques, économiques, culturelles et universitaires avec les pays anglophones, surtout avec l'Afrique du Sud, inexistantes à l'époque de l'apartheid, ce qui a, bien évidemment, des conséqueces linguistiques. Selon les estimations, de 1 à $3 \%$ des Burundais (les «lettrés » qui ont terminé leurs études secondaires ou supérieures) ont une compétence convenable en français tandis que la langue anglaise n'y est parlée que par les immigrés des pays voisins. À la différence du Rwanda qui a instauré en 2003 le trilinguisme (le kinyarwanda, le français et l'anglais sont les langues officielles du Rwanda), le danger d'anglicisation du Burundi n'est que potentiel, mais il y a des signes (relations de l'Église du Burundi, très puissante et influente, avec les pays anglophones et les pays du nord de l'Europe qui sont beaucoup plus anglophiles que francophiles ou emploi de l'anglais dans le commerce extérieur orienté de plus en plus vers les pays anglophones).

Parmi les emprunts à l'anglais fréquemment employés par les Burundais francophones, il est possible de citer boomslang «serpent arboricole Dispholidus typus kivuensis », boy (boyesse) ou groom «domestique », building « Ministère des Finances », dipping-tank « réservoir d'eau traitée pour lutter contre les tiques du bétail », wax (emprunt à l'anglais wax « cire ») « variété de pagne importée des Pays-Bas ». ${ }^{10}$ Le pseudoanglicisme taxi-man « chauffeur de taxi », utilisé dans la majorité de pays francophones en Afrique, est attesté également au Burundi.

Relativement rares sont les emprunts à d'autres langues européennes. Il s'agit surtout de mots d'origine portugaise comme capita (emprunt au portugais par le kikongo) «chef d'équipe», corossol (emprunt au portugais du Brésil) «cœur de bœuf », matabiche (emprunt au portugais matar o bicho) «pourboire». Les Burundais utilisent couramment aussi un mot latin tilapia (emprunt au latin tilapia tanganicae) «poisson perciforme endémique du Lac Tanganyika de la famille des cichlidés, herbivore, détritivore et accidentellement carnivore ».

La colonisation allemande n'a pas laissé de traces linguistiques, à l'exception du mot heller «unité monétaire créée en 1904 », "pièce de cuivre valant un centième de roupie », attestée dans la littérature burundaise de langue française.

\footnotetext{
${ }^{9}$ Paul Kagamé, président rwandais, dans son discours à l'occasion des 10 ans du génocide, a accusé la France de la responsabilté des événements tandis que Bill Clinton a été très bien accueilli pendant sa visite au Rwanda en 1998.

${ }^{10}$ Cette variété de pagne est ainsi appelée en raison de la technique d'impression à la cire.
} 
Le Burundi a été colonisé par les Belges et pour cette raison le vocabulaire du français au Burundi est beaucoup plus proche du lexique du français de Belgique que de celui de France. Les Burundais emploient fréquemment les belgicismes comme académique «universitaire », agréation "agrément donné à un acte administratif », athénée « lycée », auditoire " salle de cours à l'université », avantmidi «matinée », avant-plan «premier plan», candidature «premier cycle universitaire », chicon «endive», écolage «auto-école militaire », école gardienne "école maternelle », doubler "recommencer une classe après un échec », endéans «à l'intérieur d'une limite de temps », entièreté «totalité », minerval «frais de scolarité », par après « ensuite», roulage «circulation routière », singlet «maillot de corps », valves «tableau d'affichage », vidange « consigne ».

Certains néologismes ont été dérivés au Burundi à partir de mots empruntés : boyerie « local à l'usage du personnel domestique », murundiser « remplacer les expatriés par du personnel burundais », susuruker ou sussuruker (emprunt au kirundi gususuruka « avoir chaud ») «faire objet d'une ascension ou d'une régression sociale ou politique en fonction de son appartenance politique », ziboulateur ou zibulateur (emprunt au kirundi kuzibura « ouvrir qch qui est bouché ») « décapsuleur».

Certains mots signalés vieillis ou péjoratifs dans le français standard comme apprêter «terminer», cabaret «bistrot», cabaretier «personne qui tient un bistrot», ou accoutrement dans le sens de «vêtements » sont couramment employés dans le français au Burundi.

Le français au Burundi emploie pour la formation des néologismes les mêmes préfixes et suffixes que le français standard. Parmi les mots formés par adjonction de préfixes, on peut citer déparcher «enlever la parche (pulpe) qui enrobe les grains de café » ou postalphabétisation «phase qui suit l'alphabétisation et qui cherche à en maintenir les acquis ».

Mais c'est surtout la suffixation qu'on utilise au Burundi pour la création des mots dérivés. Outre les mots dérivés à partir de noms propres (bagazisme «politique de la Deuxième République, du président Bagaza », bagaziste « relatif $\mathrm{au}$ régime politique du président Bagaza», buyoyisme «attitude politique correspondant aux prises de position du président Buyoya », buyoyiste «partisan de la politique du président Buyoya »), on peut mentionner les mots comitard «personne faisant partie d'un comité d'organisation d'une cérémonie de mariage ou d'une fête », criseur "personne qui se trouve dans une situation matérielle ou financière difficile », déparchage "action d'enlever la pulpe qui se trouve autour des grains de café», dépulpeuse «machine qui sert à dépulper le café », kirundisation «introduction du kirundi à l'école», kirundiser «introduire le kirundi à l'école », kirundisme "expression en français calquée sur une structure linguistique rundi », lavadeur «blanchisseur», lunetté «personne qui porte des lunettes », siester «faire la sieste», sûretard «agent de la sûreté », sûreter « surveiller la population ou les individus », ventriote «homme politique qui fait passer ses intérêts personnels avant ceux de la nation ».

À cause des relations tendues entre les deux groupes principaux de la population burundaise, plusieurs néologismes ont été créés à partir de substantifs Hutu (déhutisation «passage du statut de hutu à un statut supérieur », se déhutiser « passer du statut de hutu à la caste supérieure », hutisant, hutusant ou hutiste 
« qui prend parti pour les Hutu », hutiser « mettre en place du personnel hutu dans les structures administratives et politiques », hutisme «tendance politique visant à privilégier les Hutu », Hutuland «dans la théorie de la partition du pays, partie du Burundi qui serait réservée aux Hutu») et Tutsi (détutsification « réduction du nombre du personnel tutsi dans les structures administratives et politiques », tutsification ou tutsisation «mise en place du personnel tutsi dans les structures administratives et politiques », tutsisant ou tutsiste «favorable aux Tutsi », tutsiser «mettre en place du personnel tutsi dans les structures administratives et politiques », tutsisme «tendance politique visant à privilégier les Tutsi », Tutsiland «dans la théorie de la partition du pays, partie du Burundi qui serait réservée aux Tutsi »). Les Burundais emploient fréquemment des mots dérivés à partir du nom ethnie (ethnicisation ou ethnisation « fait de donner un caractère ethnique », ethniciser ou ethniser «donner un caractère ethnique », ethniquement « du point de vue ethnique », ethnisant «qui s'appuie sur des considérations ethniques », ethnisme « tendance qui tend à favoriser la ségrégation ethnique », ethniste «personne qui favorise la ségrégation ethnique », ethnocide «extermination d'une ethnie», ethnocratie «régime politique dans lequel le pouvoir appartient à une ethnie », ethnocratique «relatif à l'ethnocratie »), rarement utilisés ou inexistants dans le français standard.

Dans le français au Burundi, on trouve plusieurs composés non attestés dans le français standard. On peut mentionner par exemple borne-fontaine «fontaine publique », caféiculteur «agriculteur spécialisé dans la culture du café », combicamping «camping-car», devin-guérisseur «médiateur entre le monde des hommes et celui des esprits », garde-bœuf «petit échassier blanc Bubulcus ibis vivant dans l'environnement des troupeaux de bovins ou des hippopotames », grisgris « amulette », ${ }^{11}$ interlacustre « qui se situe dans la Région des Grands Lacs », main-gauche «voiture dont le volant est à droite », ${ }^{12}$ mama-benz (du français employé dans la République démocratique du Congo) «femme africaine d'un certain âge, ayant réussi dans les affaires », néo-alphabète "personne récemment alphabétisée », pique-bœuf «petit oiseau Buphagus africanus au plumage gris qui vit en symbiose avec des grands herbivores et se nourrit des tiques qui les parasitent », ${ }^{13}$ radio-trottoir « rumeur », ${ }^{14}$ sans-échec ou sans-défaite « jeune tutsi des agglomérations urbaines appartenant à des bandes organisées », sous-région « subdivision de la région », taxi-vélo «bicyclette utilisée pour transporter des passagers contre rémunération », tradi-praticien «guérisseur », tribalo-terroriste «dans la terminologie uproniste, ${ }^{15}$ se dit d'un hutu qui commet des actes terroristes, en se fondant sur des considérations ethniques », vélo-bananes (kamikadze-bananes) «cycliste qui livre des bananes ».

Parmi les abréviations et acronymes, on peut citer admicom (administrateur communal) «maire », ${ }^{16}$ CADEBU «Caisse d'Épargne du Burundi », dircab

\footnotetext{
${ }^{11}$ Employé également aux Antilles.

12 Ces voitures ainsi nommées parce que le chauffeur manipule le levier de vitesses de la main gauche arrivent au Burundi clandestinement de Zambie.

${ }^{13}$ Les termes pique-bœuf et garde-bœuf sont souvent employés indifféremment pour désigner les deux espèces.

${ }^{14}$ Le substantif bujumburite désigne des rumeurs qui circulent dans la capitale.

${ }^{15}$ Partisan du parti politique UPRONA (Parti de l'Unité et du Progrès National).

${ }^{16}$ Seule la ville de Bujumbura a un maire.
} 
« directeur du cabinet », dirgal «directeur général » lona «loterie nationale », minagri « Ministère de l'Agriculture », mininter « Ministère de l'Intérieur », pafe « Police de l'Air, des Frontières et des Étrangers ».

La lexicalisation de nom propre ou de marque déposée est très productive dans le français au Burundi : coaster (selon le nom propre d'un véhicule de marque Toyota) «mini-bus», colgate «dentifrice», coleman (du nom propre de la marque) «sorte de lampe-tempête ayant un pouvoir éclairant supérieur aux lampes-tempêtes ordinaires", gatarina ou katarina "groupe organisé de malfaiteurs », ${ }^{17}$ mario "gigolo », mutoyi (d'un nom propre de lieu) «jeunes adolescentes dont les formes féminines sont déjà attestées et qui se veulent un comportement plus ou moins adulte ou qui se livrent à une forme de prostitution », ${ }_{18}$ nido «lait en poudre », nivaquine (du nom propre de la marque) « tout comprimé anti-paludéen ou antipyrétique », flavoquine (du nom propre de la marque) «tout comprimé anti-paludéen», rugombo (du nom propre de lieu) « boisson alcoolisée forte obtenue par la fermentation de bananes ».

Certaines expressions ont un sens étendu par rapport au français standard. Le mot bière désigne la bière faite à partir d'orge et de houblon, mais aussi les bières locales à base de sorgho ou de banane. Facture s'emploie indifféremment pour facture, note ou addition. Le mot colline ne désigne pas simplement un relief de terrain mais une véritable entité géographique, sociale, administrative et politique..$^{19}$ Le mot cercle ne désigne pas seulement un lieu où les membres d'une association se réunissent, mais également un lieu public où l'on peut boire, manger, se rencontrer ou se détendre». Le substantif savon désigne indifféremment le savon de toilette, la lessive ou le liquide vaisselle. Les Burundais appellent tous les Africains venant de l'Afrique de l'Ouest Sénégalais.

Par contre, le sens de certains mots est restreint par rapport au français standard. Les mèches désignent les cheveux synthétiques, le jus désignait jusqu'à une date récente le jus de maracudja. ${ }^{20}$ Les changeurs sont des personnes qui effectuent des opérations de change parallèles.

Finalement, plusieurs termes peuvent avoir un sens différent par rapport au français standard. On peut citer par exemple bock « bouteille de bière brune de 33 centilitres " «bière brune", bureau (deuxième bureau, troisième bureau) «maîtresse », divisionniste «partisan de la pratique sociale qui prend en considération l'origine ethnique ou sociale des individus », maquisard «étudiant qui n'a pas droit à une chambre universitaire et qui se fait loger chez un ami », pisteur «personne chargée d'orienter la marche d'approche dans les parcs animaliers », séminariste "participant à un séminaire », les sinistrés «ensemble de la population constituée des déplacés, des dispersés, des retournés et des rapatriés », les souplesses «les tennis », zéro « carrefour ».

En ce qui concerne la fréquence d'emploi, le contexte socio-culturel entraîne un emploi courant des mots comme malaria, pagne, don, mangue. Mais parfois il

\footnotetext{
${ }^{17}$ Il s'agit d'un emprunt de retour car le prénom kirundi Gatarina vient du prénom français Catherine.

${ }^{18}$ Les Mutoyi sont ainsi appelées parce qu'elles donnent l'impression d'avoir grandi et grossi trop vite à la manière des poulets engraissés de la coopérative agricole de Mutoyi.

${ }^{19}$ Les pays des (aux) mille collines est une dénomination courante du Burundi ainsi que du Rwanda.

${ }^{20}$ Aucun autre jus de fruit de fabrication locale n'était commercialisé au Burundi jusqu'à une date récente.
} 
est difficile d'expliquer l'origine de la fréquence plus élevée d'une terme (verbe débuter dans le français au Burundi).

\title{
5. Conclusion
}

La position du français au Burundi et dans les autres anciennes colonies belges en Afrique est moins favorable et plus vulnérable que dans les autres pays africains de langue française situés plus au Nord. Le Burundi, le Rwanda et le Congo vivent une étape de transition en matière de politique linguistique. Le Burundi a reconnu l'officialité à la langue nationale et élargit le champ de son utilisation, ce qui a un impact sur le vocabulaire du français employé dans le pays. Le français au Burundi est riche en emprunts au kirundi et au swahili et en calques par rapport à la langue nationale burundaise. Plusieurs mots ont été empruntés à l'anglais. La dérivation et la composition ont permis de créer plusieurs néologismes et assez nombreux sont aussi les particularismes sémantiques par rapport au français standard.

\section{BIBLIOGRAPHIE}

ACQUIER, Jean-Louis (1986), Le Burundi, Marseille, Parenthèses.

SHYIRAMBERE, Spiridion (1979), Le français au Rwanda et au Burundi. In : Valdman, Albert, Le français hors de France, Paris, Champion, p. 472-492.

FREY, Claude (1996), Le français au Burundi. Lexicographie et culture, Paris, EDICEF.

\section{Sites Internet}

LECLERC, Jacques : Burundi. Aménagement linguistique dans le monde [www document 6. 10. 2006], http ://www.tlfq.ulaval.ca/axl/afrique/burundi.htm Revue du Réseau des Observatoires du français contemporain en Afrique noire [www document 6. 10. 2006], http ://www.unice.fr/ILFCNRS/ofcaf/11/pays/burundi.html

\begin{abstract}
The position of French in Burundi and other former Belgian colonies in Africa is less favourable and more vulnerable than in the other African French-speaking countries situated more in the North. Burundi, Rwanda and Congo undergo a transition in the field of language policy. Burundi has awarded an official status to the national language and has extended the field of its utilisation, which has had an impact on the local French vocabulary. The French in Burundi contains abundant loan words from Kirundi and Swahili as well as many calques from the national language of Burundi. Many words have been borrowed from English. The derivation and the composition permitted to form various neologisms. In addition, we may identify numerous semantic particularities if compared to the standard French.
\end{abstract}

